\title{
Internações por Condições Sensíveis à Atenção Primária no estado do Ceará, 2010- 2014
}

\author{
Hospitalization by Conditions Sensitive to Primary Care in the state \\ of Ceará, 2010-2014
}

José Wesley dos Santos Alves', Caio Garcia Correia Sá Cavalcanti2 ${ }^{2}$ Raquel Simões Monteiro Alves $^{3}$, Priscila Chagas da Costa 4

DOI: $10.1590 / 0103-110420185418$

'Escola de Saúde Pública do Ceará (ESP/CE), Residência Integrada em Saúde - Fortaleza (CE), Brasil.

Orcid: https://orcid. org/0000-0001-90401147

wesleysa.ce@gmail.com

2 Escola de Saúde Pública do Ceará (ESP/CE),

Diretoria de Educação Profissional em Saúde Fortaleza (CE), Brasil. Orcid: https://orcid. org/0000-0002-00971507

caio.cavalcanti2011@gmail. com

3 Instituto Federal de Educação, Ciência e Tecnologia do Ceará (IFCE)

- Crateús (CE), Brasil. Orcid: https://orcid. org/0000-0003-42545845

raquelsmnutrigmail.com

${ }^{4}$ Escola de Saúde Pública do Ceará (ESP/CE), Centro de Educação Permanente em Vigilância da Saúde Fortaleza (CE), Brasil.

Orcid: https://orcid. org/0000-0002-0979-

1916

enfapriscilacosta@hotmail. com
RESUMO Este artigo objetivou descrever o perfil das Internações por Condições Sensíveis à Atenção Primária (ICSAP) nas 22 Regiões de Saúde do Ceará, através de um estudo quantitativo e transversal sobre as hospitalizações ocorridas entre 2010 e 2014. Houve redução das ICSAP nas regiões, com exceção de duas. Na maioria, a ampliação da cobertura da Estratégia Saúde da Família gerou redução na proporção de ICSAP. Entretanto, é necessário um compromisso mais contundente com relação à sua qualidade, à consolidação do processo de regionalização e ao desenvolvimento de ações intersetoriais que impactem em outros determinantes de saúde.

PALAVRAS-CHAVe Hospitalização. Sistema Único de Saúde. Atenção Primária à Saúde. Regionalização.

ABSTRACT This article aimed to describe the profile of Hospitalizations due to Conditions Sensitive to Primary Care (ICSAP) in the 22 Health Regions of Ceará, through a quantitative and crosssectional study on hospitalizations between 2010 and 2014. There was a reduction of ICSAP in the regions, except for two. In the majority, the expansion of the coverage of the Family Health Strategy has generated a reduction in the proportion of ICSAP. However, a overwhelming commitment to quality is required, to the consolidation of the regionalization process and to the development of intersectoral actions that impact on other health determinants.

KEYWORDS Hospitalization. Unified Health System. Primary Health Care. Regional health planning. 


\section{Introdução}

A Atenção Primária à Saúde (APS) representa o componente de um sistema de saúde que permite tanto a entrada prioritária de pessoas aos serviços assistenciais como o acompanhamento longitudinal de sujeitos saudáveis ou não em seu ambiente comunitário. Ela deve fazer o papel de coordenar e integrar os demais pontos de atenção à saúde, racionalizar os custos do sistema, abordar os problemas sanitários mais comuns da comunidade, com responsabilidade pelo acesso, pela prevenção, pelo tratamento, pela cura e pela reabilitação dos usuários através de processos de trabalho em equipe, visando à máxima condição de saúde e bem-estar dos sujeitos ${ }^{1}$.

Embora seja visto durante a história de construção do Sistema Único de Saúde (SUS) um crescimento da oferta de serviços de APS que indique uma ampliação de acesso, ainda estão postos alguns desafios para atendimento das necessidades de saúde da população, a exemplo da fragmentação das políticas e dos programas de saúde ${ }^{2,3}$. Deste modo, é imprescindível a estruturação de uma rede solidária e regionalizada de ações e serviços que qualifiquem o processo de gestão ${ }^{3}$.

Atualmente, tomam destaque discussões sobre a implantação de Redes de Atenção à Saúde (RAS) ${ }^{4}$, que têm posicionado a APS como coordenadora do sistema de saúde, de modo a assegurar a integralidade e a continuidade do cuidado com vistas à resolubilidade dos problemas sanitários ${ }^{5}$.

Para auxiliar na avaliação dos sistemas de saúde, as Internações por Condições Sensíveis à Atenção Primária (ICSAP) podem servir como indicador indireto de qualidade do acesso e desempenho da APS, revelando a efetividade das ações de saúde oferecidas nesse níveli,6. A fundamentação para essa questão reside na hipótese de que uma elevada resolubilidade na atenção primária deveria repercutir em uma redução dos ingressos hospitalares, através do aumento das medidas preventivas e da otimização dos tratamentos ambulatoriais $\mathbf{s}^{\mathbf{3}, \mathbf{8}}$.
Em 2008, a Secretaria de Atenção à Saúde (SAS), do Ministério da Saúde (MS), publicou oficialmente a Portaria SAS/MS no 221, de 17 de abril de 2008, que traz a Lista Brasileira de Internações por Condições Sensíveis à Atenção Primária, relacionada por grupos de causas de internações e diagnósticos, de acordo com a Décima Revisão da Classificação Internacional de Doenças (CID-10), a qual pode ser aplicada para avaliar o desempenho do sistema de saúde nos âmbitos nacional, estadual e municipal 9 .

No estado do Ceará, o sistema de saúde está estruturado em 22 Regiões de Saúde (RS) e 5 Macrorregiões de Saúde ${ }^{10}$. As RS são agrupamentos de municípios limítrofes, demarcados a partir de identidades culturais, econômicas e sociais e de redes de comunicação e transporte compartilhadas, visando a integrar a organização, o planejamento e a execução de ações e serviços de saúde ${ }^{11}$. A APS está organizada em cada município; os serviços especializados de média complexidade são pactuados entre o estado e as RS; as macrorregiões estão definidas pela oferta de serviços especializados de alta complexidade e alto custo ${ }^{12}$.

Considerando a ênfase na diretriz da regionalização na atual organização do SUS e a importância da APS nesse contexto ${ }^{5,11}$, este estudo tem o objetivo de descrever o perfil das ICSAP no estado do Ceará, entre os anos de 2010 a 2014, de modo a subsidiar o planejamento de ações de saúde voltadas para a organização dos sistemas regionais de saúde.

\section{Métodos}

Trata-se de um estudo quantitativo e transversal sobre as ICSAP de pacientes residentes nas RS do estado do Ceará, pagas pelo SUS, ocorridas no período de 2010 a 2014.

As informações sobre as internações foram obtidas no Sistema de Informações Hospitalares do Sistema Único de Saúde (SIH/SUS), disponibilizado no sítio eletrônico do Departamento de Informática do SUS (Datasus) ${ }^{\mathbf{1 3}}$, cuja fonte de dados foi 
a Autorização de Internação Hospitalar (AIH/SUS).

Foram também levantadas informações de cobertura da população por Equipes de Saúde da Família (EqSF), a partir do Sistema de Informação da Atenção Básica (Siab) do Datasus ${ }^{\mathbf{1 4}}$, ainda utilizado àquela época e ora progressivamente substituído pelo e-SUS Atenção Básica (e-SUS AB). O Siab teve como objetivo agregar, armazenar e processar as informações relacionadas à APS utilizando como estratégia central a Estratégia Saúde da Família (ESF) ${ }^{\mathbf{1 5}}$. Para o cálculo da cobertura populacional da ESF foi considerado o número de EqSF e a média de 3.450 pessoas (média de pessoas acompanhadas por uma EqSF) ${ }^{\mathbf{1 6}}$, com limitador de $100 \%$ de cobertura. No denominador, utilizou-se a estimativa populacional em cada ano analisado.

O diagnóstico principal das internações registrado no SIH/SUS foi adotado como causa de internação, de acordo com a CID-10. Excluídos os partos (CID-10: O80 a O84), por representarem desfecho natural à gestação, as internações foram classificadas como Condições Sensíveis à Atenção Primária (CSAP), de acordo com a Lista Brasileira publicada como anexo da Portaria SAS/MS n ${ }^{0}$ 221/20089 ${ }^{9}$. As internações gerais compreenderam o somatório das ICSAP e demais internações.

Considerou-se o ano de atendimento da AIH/ SUS para definir a temporalidade do evento. Dados referentes ao sexo foram assumidos sem modificações. Os subgrupos etários seguiram a mesma categorização do Datasus, porém, optou-se por agregar os dados do subgrupo menores de 1 ano ao subgrupo com idade de 1 a 4 anos, gerando um subgrupo de 0 a 4 anos, com a finalidade de igualar os dados etários ao padrão de dados de projeção populacional, facilitando o processo de análise. As RS foram definidas segundo o mapa da regionalização estabelecido no Plano Diretor de Regionalização (PDR) do Ceará, totalizando 22 regiões $^{10}$.

Os dados foram inseridos em uma planilha eletrônica do programa Microsoft Excel 2013. Foram calculados os coeficientes de ICSAP ( ${ }^{\circ}$ ICSAP de pacientes residentes nas RS/ população das RS no ano x 1.000 habitantes) e de internações gerais ( $\mathrm{n}^{\circ}$ internações gerais de pacientes residentes nas RS/população das RS no ano x 1.000 habitantes), no período de 2010 a 2014. Calcularam-se, também, as variações percentuais da frequência de ICSAP e dos coeficientes de ICSAP e de internações gerais entre os anos de 2014 e 2010.

Outra análise realizada foi a relação entre a cobertura populacional de EqSF e a participação percentual das ICSAP no total das internações de pacientes residentes, segundo RS.

Os dados populacionais utilizados foram os do Censo Demográfico 2010 e as estimativas anuais enviadas ao Tribunal de Contas da União (TCU), ambos disponíveis no sítio eletrônico do Instituto Brasileiro de Geografia e Estatística (IBGE) ${ }^{17}$.

Este estudo não foi submetido a Comitê de Ética em Pesquisa por utilizar dados secundários de domínio público. Entretanto, foram respeitadas as normas vigentes no Brasil relacionadas à ética na pesquisa com seres humanos, constantes na Resolução $\mathrm{n}^{\circ} 466$, de 12 de dezembro de 2012, da Comissão Nacional de Ética em Pesquisa (Conep). Os dados foram analisados especificamente para esta pesquisa, sem qualquer identificação individual.

\section{Resultados}

Conforme os dados do SIH/SUS, houve variação de $-8,6 \%$ nas internações gerais entre os anos de 2010 e 2014 no estado do Ceará. Houve variação percentual negativa nas internações gerais em todas as RS, exceto para Maracanaú (7,7\%) e Russas (4,8\%). As maiores reduções ocorreram nas RS de Iguatu (-31,3\%) e Sobral $(-24,9 \%)^{13}$.

Houve, também, redução na frequência das ICSAP no estado do Ceará (tabela 1). Semelhantemente ao que ocorreu com as internações gerais, verificou-se aumento da variação percentual de ICSAP nas RS de Maracanaú e Russas, tendo esta apresentado uma ampliação superior a 80\%. Iguatu e Sobral lideraram as reduções. 
Tabela 1. Número de Internações por Condições Sensíveis à Atenção Primária (ICSAP) registradas no SUS, segundo RS de residência do paciente no estado do Ceará, 2010 a 2014

\begin{tabular}{|c|c|c|c|c|c|c|}
\hline \multirow{2}{*}{ Região de Saúde (RS) } & \multicolumn{5}{|c|}{ Frequência de internação paciente residente por CSAP } & \multirow{2}{*}{$\begin{array}{l}\text { Variação \% } \\
2014 / 2010\end{array}$} \\
\hline & 2010 & 2011 & 2012 & 2013 & 2014 & \\
\hline 1a RS Fortaleza & 27.060 & 28.162 & 24.460 & 27.617 & 26.213 & $-3,1 \%$ \\
\hline 2a RS Caucaia & 4.416 & 4.581 & 3.979 & 4.344 & 3.941 & $-10,8 \%$ \\
\hline 3ạ RS Maracanaú & 3.929 & 4.004 & 3.316 & 4.091 & 4.047 & $3,0 \%$ \\
\hline 4a RS Baturité & 2.029 & 1.970 & 1.343 & 1.393 & 1.211 & $-40,3 \%$ \\
\hline 5a RS Canindé & 2.778 & 2.743 & 2.128 & 2.410 & 1.976 & $-28,9 \%$ \\
\hline 6a RS Itapipoca & 2.812 & 2.928 & 2.863 & 2.746 & 2.445 & $-13,1 \%$ \\
\hline 7a RS Aracati & 1.184 & 958 & 811 & 959 & 842 & $-28,9 \%$ \\
\hline 8ミ RS Quixadá & 3.764 & 3.823 & 3.041 & 3.565 & 2.985 & $-20,7 \%$ \\
\hline 9a RS Russas & 2.740 & 3.595 & 3.862 & 4.886 & 4.967 & $81,3 \%$ \\
\hline 10a RS Limoeiro do Norte & 2.909 & 3.745 & 3.172 & 3.801 & 2.834 & $-2,6 \%$ \\
\hline 11- RS Sobral & 8.720 & 8.180 & 5.874 & 6.334 & 5.169 & $-40,7 \%$ \\
\hline 12 RS Acaraú & 1.955 & 2.291 & 1.908 & 1.811 & 1.669 & $-14,6 \%$ \\
\hline 13르 RS Tianguá & 4.644 & 4.466 & 3.460 & 4.137 & 3.587 & $-22,8 \%$ \\
\hline 14ạ RS Tauá & 2.937 & 2.561 & 2.083 & 2.457 & 2.205 & $-24,9 \%$ \\
\hline 15 RS Crateús & 4.732 & 4.528 & 4.378 & 5.493 & 4.362 & $-7,8 \%$ \\
\hline 16a RS Camocim & 1.892 & 1.679 & 1.355 & 1.445 & 1.316 & $-30,4 \%$ \\
\hline 17a RS Icó & 3.834 & 3.350 & 2.388 & 3.920 & 3.372 & $-12,1 \%$ \\
\hline 18’ RS Iguatu & 6.996 & 6.635 & 5.322 & 5.090 & 4.033 & $-42,4 \%$ \\
\hline 19a RS Brejo Santo & 6.124 & 6.534 & 6.433 & 5.903 & 5.268 & $-14,0 \%$ \\
\hline 20a RS Crato & 7.743 & 7.512 & 6.313 & 6.510 & 5.204 & $-32,8 \%$ \\
\hline 21a RS Juazeiro do Norte & 6.001 & 5.360 & 4.633 & 4.402 & 5.649 & $-5,9 \%$ \\
\hline 22a RS Cascavel & 2.545 & 2.718 & 2.097 & 2.718 & 2.059 & $-19,1 \%$ \\
\hline Estado do Ceará & 111.744 & 112.323 & 95.219 & 106.032 & 95.354 & $-14,7 \%$ \\
\hline
\end{tabular}

Fonte: SIH/SUS13

Quanto aos coeficientes de ICSAP e de internações gerais (tabela 2), observou-se que o estado do Ceará apresentou redução percentual para ambos entre 2010 e 2014. As RS de Sobral e Iguatu apresentaram as maiores reduções nos coeficientes, enquanto em Russas e Maracanaú verificaram-se os maiores aumentos percentuais. Apesar das elevações no coeficiente por internações gerais e em números absolutos nos dois tipos de internação, a RS de Maracanaú demonstrou discreta diminuição no coeficiente de ICSAP. 
Tabela 2. Coeficientes de internação (por mil habitantes) por Condições Sensíveis à Atenção Primária (CSAP) e por internações gerais, segundo RS de residência do paciente no estado do Ceará, 2010 a 2014

\begin{tabular}{|c|c|c|c|c|c|c|c|c|c|c|c|c|}
\hline \multirow{3}{*}{ Região de Saúde (RS) } & \multicolumn{10}{|c|}{ Coeficiente de internação por 1.000 habitantes } & \multirow{2}{*}{\multicolumn{2}{|c|}{$\begin{array}{l}\text { Variação \% } \\
2014 / 2010\end{array}$}} \\
\hline & \multicolumn{2}{|c|}{2010} & \multicolumn{2}{|c|}{2011} & \multicolumn{2}{|c|}{2012} & \multicolumn{2}{|c|}{2013} & \multicolumn{2}{|c|}{2014} & & \\
\hline & CSAP & Geral & CSAP & Geral & CSAP & Geral & CSAP & Geral & CSAP & Geral & CSAP & Geral \\
\hline 1a RS Fortaleza & 10,4 & 56,0 & 10,7 & 56,1 & 9,2 & 51,7 & 10,2 & 54,4 & 9,6 & 51,1 & $-7,8 \%$ & $-8,8 \%$ \\
\hline 2a RS Caucaia & 7,8 & 46,0 & 8,0 & 45,9 & 6,9 & 40,5 & 7,3 & 46,1 & 6,6 & 42,6 & $-16,2 \%$ & $-7,6 \%$ \\
\hline 3a RS Maracanaú & 8,0 & 48,1 & 8,0 & 46,8 & 6,6 & 43,6 & 7,9 & 47,3 & 7,7 & 48,8 & $-3,0 \%$ & $1,4 \%$ \\
\hline 4a RS Baturité & 15,2 & 63,8 & 14,7 & 61,6 & 10,0 & 49,8 & 10,2 & 51,0 & 8,8 & 52,7 & $-42,1 \%$ & $-17,3 \%$ \\
\hline 5a RS Canindé & 14,2 & 53,9 & 13,9 & 52,2 & 10,7 & 47,8 & 11,9 & 48,2 & 9,7 & 47,8 & $-31,5 \%$ & $-11,3 \%$ \\
\hline 6a RS Itapipoca & 10,3 & 52,7 & 10,6 & 53,8 & 10,3 & 53,1 & 9,6 & 50,5 & 8,5 & 49,2 & $-17,9 \%$ & $-6,7 \%$ \\
\hline 7a RS Aracati & 10,8 & 49,8 & 8,7 & 48,0 & 7,3 & 41,7 & 8,4 & 45,9 & 7,3 & 44,0 & $-32,1 \%$ & $-11,5 \%$ \\
\hline 8a RS Quixadá & 12,3 & 53,9 & 12,4 & 56,2 & 9,8 & 51,4 & 11,3 & 52,6 & 9,4 & 48,4 & $-23,7 \%$ & $-10,2 \%$ \\
\hline 9a RS Russas & 14,4 & 72,5 & 18,7 & 67,9 & 20,0 & 66,6 & 24,9 & 68,6 & 25,2 & 73,7 & $75,8 \%$ & $1,6 \%$ \\
\hline 10a RS Limoeiro do Norte & 13,4 & 54,0 & 17,2 & 56,3 & 14,5 & 53,2 & 17,1 & 60,4 & 12,7 & 52,1 & $-5,5 \%$ & $-3,5 \%$ \\
\hline 11a RS Sobral & 14,3 & 61,4 & 13,3 & 60,9 & 9,5 & 54,2 & 10,1 & 52,1 & 8,2 & 44,2 & $-43,2 \%$ & $-28,0 \%$ \\
\hline 12a RS Acaraú & 9,3 & 46,2 & 10,7 & 46,9 & 8,8 & 43,1 & 8,2 & 39,5 & 7,5 & 36,8 & $-19,2 \%$ & $-20,4 \%$ \\
\hline 13ā RS Tianguá & 15,7 & 61,7 & 15,0 & 60,1 & 11,5 & 54,7 & 13,5 & 52,1 & 11,6 & 51,0 & $-26,2 \%$ & $-17,3 \%$ \\
\hline 14ㄹ RS Tauá & 26,5 & 75,6 & 23,0 & 65,7 & 18,7 & 63,5 & 21,7 & 61,9 & 19,4 & 65,5 & $-26,7 \%$ & $-13,4 \%$ \\
\hline 15a RS Crateús & 16,3 & 56,1 & 15,6 & 52,0 & 15,0 & 53,5 & 18,6 & 55,1 & 14,7 & 49,0 & $-9,5 \%$ & $-12,6 \%$ \\
\hline 16a RS Camocim & 12,6 & 51,3 & 11,2 & 49,3 & 9,0 & 42,7 & 9,4 & 40,5 & 8,5 & 38,1 & $-32,4 \%$ & $-25,7 \%$ \\
\hline 17ạ RS Icó & 22,8 & 61,7 & 19,9 & 61,0 & 14,2 & 46,4 & 22,9 & 59,4 & 19,7 & 56,1 & $-13,6 \%$ & $-9,1 \%$ \\
\hline 18a RS Iguatu & 22,8 & 63,8 & 21,5 & 61,5 & 17,2 & 50,9 & 16,1 & 48,3 & 12,7 & 42,4 & $-44,2 \%$ & $-33,5 \%$ \\
\hline 19a RS Brejo Santo & 29,8 & 80,2 & 31,7 & 84,3 & 31,0 & 79,4 & 28,0 & 73,4 & 24,9 & 68,7 & $-16,6 \%$ & $-14,4 \%$ \\
\hline 20ミ RS Crato & 23,6 & 78,9 & 22,7 & 78,1 & 19,0 & 70,6 & 19,2 & 66,8 & 15,3 & 62,6 & $-35,2 \%$ & $-20,6 \%$ \\
\hline 21a RS Juazeiro do Norte & 15,1 & 66,4 & 13,4 & 60,5 & 11,5 & 56,7 & 10,7 & 50,9 & 13,6 & 59,5 & $-10,0 \%$ & $-10,4 \%$ \\
\hline 22ㄹ RS Cascavel & 8,7 & 45,9 & 9,1 & 46,0 & 6,9 & 40,3 & 8,7 & 45,1 & 6,5 & 40,4 & $-24,5 \%$ & $-12,0 \%$ \\
\hline Estado do Ceará & 13,2 & 57,5 & 13,2 & 56,8 & 11,1 & 51,8 & 12,1 & 53,0 & 10,8 & 50,3 & $-18,4 \%$ & $-12,6 \%$ \\
\hline
\end{tabular}

Fonte: SIH/SUS (Datasus) ${ }^{\mathbf{1 3}}$ e IBGE17.

Nos anos de 2010 e 2014, as ICSAP compreenderam, respectivamente, $23,0 \%$ e $21,4 \%$ das internações gerais no Ceará. A maior proporção ocorrida no estado foi em 2011 (23,2\%). Em termos percentuais, houve variação positiva entre as proporções de 2014 em relação a 2010 nas RS de Russas (14,5\%), Crateús (1,1\%), Acaraú (0,3\%), Fortaleza (0,2\%) e Juazeiro do Norte (0,1\%). As demais RS tiveram uma redução no percentual, com destaque para Baturité (-7,2\%), Canindé $(-6,0 \%)$ e Iguatu $(-5,7 \%)$.
Analisou-se, também, a relação entre a cobertura pelas EqSF e o percentual de ICSAP no tocante às internações gerais (gráfico 1). Observou-se em todas as RS ampliação da cobertura de EqSF entre 2010 e 2014, com exceção daquelas que já haviam atingido $100 \%$ nos dois anos referidos. Destaca-se a baixa cobertura de EqSF verificada na RS de Fortaleza, ainda que tenha demonstrado um aumento discreto nesse indicador em 2014.

Apesar da ampliação da cobertura de EqSF, 
a RS de Russas apresentou importante elevação da proporção de ICSAP, contrariando as expectativas de que a ampliação da cobertura da APS corrobora para reduzir o número de hospitalizações por ICSAP. Situação semelhante foi observada nas RS de Fortaleza, Acaraú, Crateús e Juazeiro do Norte, porém, em uma amplitude menor. Isso parece demonstrar que o aumento da cobertura de EqSF não repercutiu em um aprimoramento no acesso aos serviços, bem como nos processos assistenciais primários ofertados nessas RS. Nas demais RS, a ampliação ou a manutenção de $100 \%$ da cobertura de EqSF foi acompanhada de uma redução nas ICSAP.

Gráfico 1. Cobertura da população por Equipes de Saúde da Família (EqSF) e participação das Internações por Condições Sensíveis à Atenção Primária (ICSAP) no total das internações de pacientes residentes, segundo RS. Ceará, 2010 e 2014

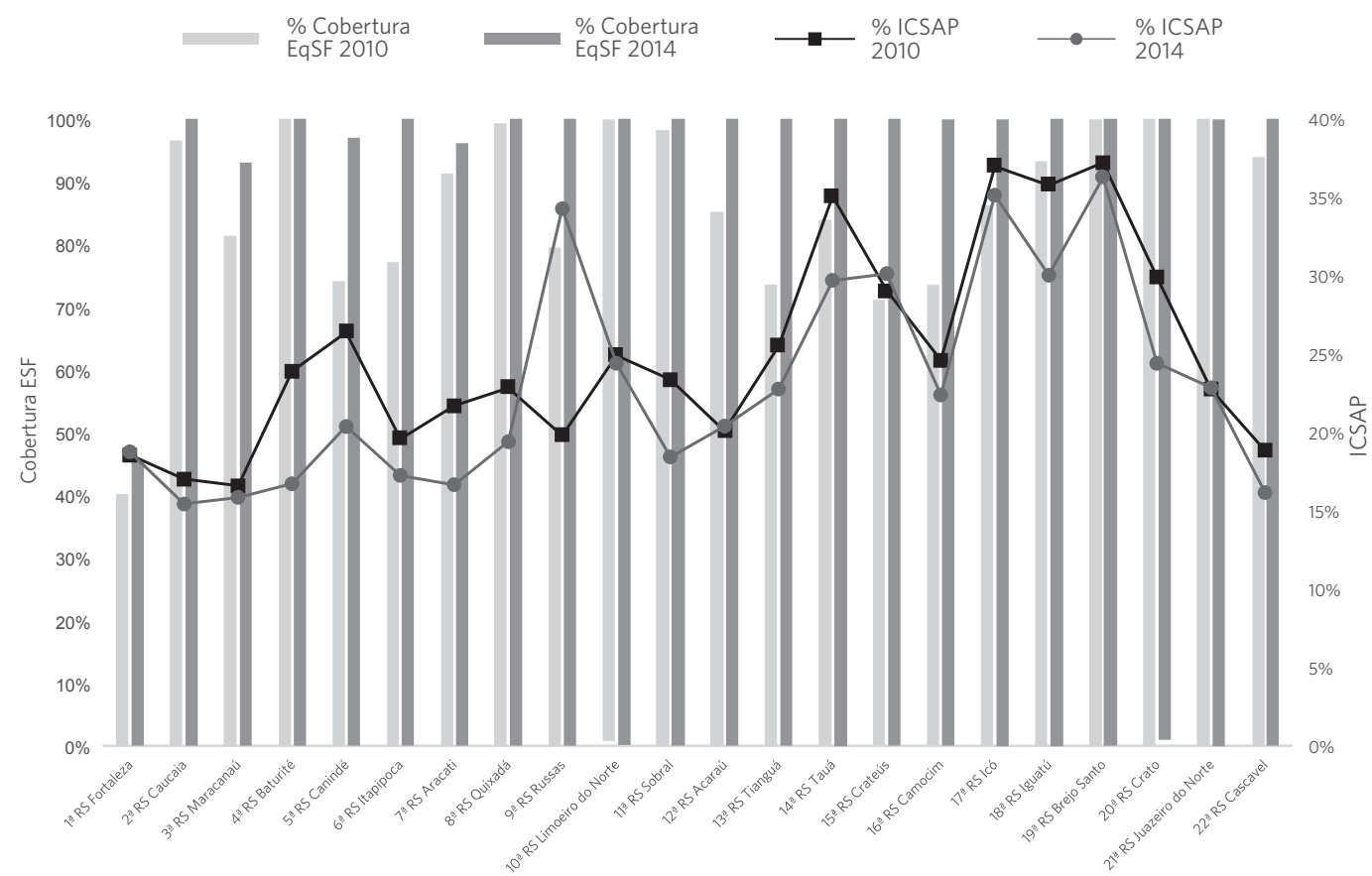

Fonte: Siab/MS14-15, SIH/SUS (Datasus) ${ }^{\mathbf{1 3}}$ e IBGE17.

No que diz respeito às variações percentuais por grupo de causas de ICSAP (tabela 3), observou-se redução na maioria dos grupos no estado. Houve aumento da frequência de internações no Ceará, os maiores ocorrendo para infecções de pele e do tecido subcutâneo e doenças relacionadas ao pré-natal e ao parto. Os demais incrementos foram observados nas doenças pulmonares, cerebrovasculares e epilepsias. As reduções mais significativas foram para úlceras gastrointestinais, anemia, asma, gastroenterites infecciosas e complicações e hipertensão. 
Tabela 3. Variação percentual na frequência de Internações por Condições Sensíveis à Atenção Primária (ICSAP), segundo grupo de causas e Região de Saúde de residência no estado do Ceará, 2014 em relação a 2010

\begin{tabular}{|c|c|c|c|c|c|c|c|c|}
\hline \multirow{2}{*}{ Grupo de Causas (CSAP) } & \multicolumn{8}{|c|}{ Região de Saúde (RS) } \\
\hline & Fortaleza & \multicolumn{2}{|c|}{ Caucaia } & Maracanaú & Baturité & Canindé & Itapipoca & Aracati \\
\hline $\begin{array}{l}\text { 1. Doenças preveníveis p/ imunização e cond. } \\
\text { sensíveis }\end{array}$ & $15,1 \%$ & \multicolumn{2}{|c|}{$42,9 \%$} & $-46,2 \%$ & $100,0 \%$ & $62,5 \%$ & $50,0 \%$ & $-80,0 \%$ \\
\hline 2. Gastroenterites infecciosas e complicações & $-33,0 \%$ & $-46,0 \%$ & \multicolumn{2}{|c|}{$-22,1 \%$} & $-62,8 \%$ & $-36,0 \%$ & $-29,5 \%$ & $-36,0 \%$ \\
\hline 3. Anemia & $-65,6 \%$ & $-61,0 \%$ & \multicolumn{2}{|c|}{$-69,6 \%$} & $-100,0 \%$ & $-6,6 \%$ & $-35,9 \%$ & $-16,7 \%$ \\
\hline 4. Deficiências nutricionais & $-50,9 \%$ & \multicolumn{2}{|c|}{$-50,9 \%$} & $-53,1 \%$ & $-16,7 \%$ & $-12,5 \%$ & $57,1 \%$ & $-14,3 \%$ \\
\hline 5. Infecções de ouvido, nariz e garganta. & $-19,2 \%$ & $-57,8 \%$ & & $6 \%$ & $0,0 \%$ & $233,3 \%$ & $160,0 \%$ & $-50,0 \%$ \\
\hline 6. Pneumonias bacterianas & $11,6 \%$ & & & ,7\% & $-33,0 \%$ & $-37,7 \%$ & $-19,4 \%$ & $-7,7 \%$ \\
\hline 7. Asma & $-28,1 \%$ & -26 & & $6 \%$ & $-68,1 \%$ & $-55,6 \%$ & $-50,0 \%$ & $-65,8 \%$ \\
\hline 8. Doenças pulmonares & $33,0 \%$ & & & $2 \%$ & $35,4 \%$ & $-1,9 \%$ & $375,0 \%$ & $-56,3 \%$ \\
\hline 9. Hipertensão & $-67,2 \%$ & & & $0 \%$ & $-87,5 \%$ & $25,7 \%$ & $-76,3 \%$ & $-30,8 \%$ \\
\hline 10. Angina & & & DADOS NÃ & DISPONÍV & /EIS NO SIH/S & US - DATASL & & \\
\hline 11. Insuficiência cardíaca & $-6,2 \%$ & & $7 \%$ & $9 \%$ & $-34,2 \%$ & $-15,6 \%$ & $-41,3 \%$ & $-23,9 \%$ \\
\hline 12. Doenças cerebrovasculares & $63,7 \%$ & & $8 \%$ & $6 \%$ & $24,7 \%$ & $-31,8 \%$ & $19,8 \%$ & $-31,8 \%$ \\
\hline 13. Diabetes mellitus & $-23,9 \%$ & & & $8 \%$ & $-57,0 \%$ & $30,8 \%$ & $10,1 \%$ & $-10,6 \%$ \\
\hline 14. Epilepsias & $82,8 \%$ & & & $9 \%$ & $27,3 \%$ & $75,0 \%$ & $54,5 \%$ & $-25,0 \%$ \\
\hline 15. Infecção no rim e trato urinário & $0,5 \%$ & & $6 \%$ & $0 \%$ & $-52,3 \%$ & $-22,0 \%$ & $-32,6 \%$ & $-47,3 \%$ \\
\hline 16. Infecção da pele e tecido subcutâneo & $46,8 \%$ & & & $3 \%$ & $-36,1 \%$ & $-61,7 \%$ & $314,3 \%$ & $0,0 \%$ \\
\hline $\begin{array}{l}\text { 17. Doença inflamatória órgãos pélvicos fe- } \\
\text { mininos }\end{array}$ & $-10,6 \%$ & -33 & & $5 \%$ & $-53,6 \%$ & $-41,9 \%$ & $30,8 \%$ & $-79,5 \%$ \\
\hline 18. Úlcera gastrointestinal & $-42,5 \%$ & & & $3 \%$ & $0,0 \%$ & $-60,0 \%$ & $-37,5 \%$ & $-75,0 \%$ \\
\hline 19. Doenças relacionadas ao pré-natal e parto & $23,0 \%$ & & & $7 \%$ & $0,0 \%$ & $0,0 \%$ & $146,7 \%$ & * \\
\hline & & & & Região de & Saúde (RS) & & & \\
\hline Grupo de Causas (CSAP) & Quixadá & Russas & $\begin{array}{l}\text { Limoeiro } \\
\text { do Norte }\end{array}$ & Sobral & Acaraú & Tianguá & Tauá & Crateús \\
\hline $\begin{array}{l}\text { 1. Doenças preveníveis } \mathrm{p} / \text { imunização e } \\
\text { cond. sensíveis }\end{array}$ & $122,2 \%$ & $-52,9 \%$ & $116,7 \%$ & $93,5 \%$ & $53,8 \%$ & $6,7 \%$ & $100,0 \%$ & $-66,7 \%$ \\
\hline $\begin{array}{l}\text { 2. Gastroenterites infecciosas e compli- } \\
\text { cações }\end{array}$ & $-28,4 \%$ & $69,1 \%$ & $3,2 \%$ & $-63,8 \%$ & $-25,3 \%$ & $-50,4 \%$ & $-53,1 \%$ & $-6,3 \%$ \\
\hline 3. Anemia & $8,6 \%$ & $825,0 \%$ & $78,6 \%$ & $-95,7 \%$ & $-50,0 \%$ & $6,1 \%$ & * & $-32,1 \%$ \\
\hline 4. Deficiências nutricionais & $-40,7 \%$ & $52,6 \%$ & $-88,9 \%$ & $61,0 \%$ & $-71,4 \%$ & $-80,0 \%$ & * & $-58,8 \%$ \\
\hline 5. Infecções de ouvido, nariz e garganta. & $-87,1 \%$ & $208,2 \%$ & $-25,7 \%$ & $-55,1 \%$ & $0,0 \%$ & $2,2 \%$ & $683,3 \%$ & $-25,0 \%$ \\
\hline 6. Pneumonias bacterianas & $-4,9 \%$ & $123,8 \%$ & $-9,4 \%$ & $-27,7 \%$ & $39,6 \%$ & $2,1 \%$ & $72,6 \%$ & $15,9 \%$ \\
\hline 7. Asma & $-62,4 \%$ & $-5,9 \%$ & $-51,0 \%$ & $-48,1 \%$ & $-62,3 \%$ & $-67,2 \%$ & $-75,5 \%$ & $-55,5 \%$ \\
\hline 8. Doenças pulmonares & $-22,5 \%$ & $158,9 \%$ & $79,2 \%$ & $-36,8 \%$ & $-44,7 \%$ & $-32,9 \%$ & $370,0 \%$ & $0,0 \%$ \\
\hline 9. Hipertensão & $-52,6 \%$ & $53,0 \%$ & $-46,6 \%$ & $-92,8 \%$ & $-87,5 \%$ & $-73,3 \%$ & $-63,6 \%$ & $18,6 \%$ \\
\hline 10. Angina & & & ADOS NÃO & SPONÍVEI & IS NO SIH/SU & IS - DATASUS & & \\
\hline 11. Insuficiência cardíaca & $-22,7 \%$ & $21,0 \%$ & $-0,6 \%$ & $-14,6 \%$ & $-4,0 \%$ & $-35,5 \%$ & $-37,4 \%$ & $-23,9 \%$ \\
\hline 12. Doenças cerebrovasculares & $13,9 \%$ & $5,5 \%$ & $21,5 \%$ & $-22,7 \%$ & $-20,9 \%$ & $10,4 \%$ & $-8,0 \%$ & $-20,0 \%$ \\
\hline 13. Diabetes mellitus & $-13,0 \%$ & $43,5 \%$ & $-22,1 \%$ & $-50,5 \%$ & $-46,5 \%$ & $-21,8 \%$ & $-68,2 \%$ & $-5,1 \%$ \\
\hline
\end{tabular}


Tabela 3. (cont.)

\begin{tabular}{|c|c|c|c|c|c|c|c|c|}
\hline 14. Epilepsias & $-7,5 \%$ & $167,9 \%$ & $95,8 \%$ & $-41,8 \%$ & $11,1 \%$ & $-44,4 \%$ & $-47,8 \%$ & $15,4 \%$ \\
\hline 15. Infecção no rim e trato urinário & $-13,4 \%$ & $95,8 \%$ & $-14,9 \%$ & $-51,1 \%$ & $-23,1 \%$ & $-4,4 \%$ & $-27,4 \%$ & $-4,8 \%$ \\
\hline 16. Infecção da pele e tecido subcutâneo & $-62,9 \%$ & $272,1 \%$ & $4,3 \%$ & $78,8 \%$ & $31,9 \%$ & $140,5 \%$ & $223,8 \%$ & $23,1 \%$ \\
\hline $\begin{array}{l}\text { 17. Doença inflamatória órgãos pélvicos } \\
\text { femininos }\end{array}$ & $20,9 \%$ & $47,5 \%$ & $-50,0 \%$ & $-45,3 \%$ & $-24,0 \%$ & $43,1 \%$ & $200,0 \%$ & $-34,5 \%$ \\
\hline 18. Úlcera gastrointestinal & $47,1 \%$ & $-57,5 \%$ & $-35,3 \%$ & $-34,6 \%$ & $-66,7 \%$ & $-9,1 \%$ & $180,0 \%$ & $-33,3 \%$ \\
\hline \multirow[t]{2}{*}{$\begin{array}{l}\text { 19. Doenças relacionadas ao pré-natal e } \\
\text { parto }\end{array}$} & $200,0 \%$ & $100,0 \%$ & $300,0 \%$ & $97,3 \%$ & $150,0 \%$ & $-75,0 \%$ & $-100,0 \%$ & $425,0 \%$ \\
\hline & \multicolumn{7}{|c|}{ Região de Saúde (RS) } & \\
\hline Grupo de Causas (CSAP) & Camocim & Icó & Iguatu & $\begin{array}{l}\text { Brejo } \\
\text { Santo }\end{array}$ & Crato & $\begin{array}{l}\text { Juazeiro } \\
\text { do Norte }\end{array}$ & Cascavel & $\begin{array}{l}\text { Estado do } \\
\text { Ceará }\end{array}$ \\
\hline $\begin{array}{l}\text { 1. Doenças preveníveis } \mathrm{p} / \text { imunização e } \\
\text { cond. sensíveis }\end{array}$ & $37,5 \%$ & $63,6 \%$ & $100,0 \%$ & $74,3 \%$ & $-60,4 \%$ & $-15,6 \%$ & $80,0 \%$ & $-1,2 \%$ \\
\hline $\begin{array}{l}\text { 2. Gastroenterites infecciosas e compli- } \\
\text { cações }\end{array}$ & $-45,7 \%$ & $-28,3 \%$ & $-64,5 \%$ & $-26,4 \%$ & $-40,3 \%$ & $-4,5 \%$ & $-44,4 \%$ & $-34,5 \%$ \\
\hline 3. Anemia & $-77,8 \%$ & $-59,1 \%$ & $-79,9 \%$ & $-28,2 \%$ & $-82,6 \%$ & $-61,1 \%$ & $-80,5 \%$ & $-42,0 \%$ \\
\hline 4. Deficiências nutricionais & $350,0 \%$ & $-19,0 \%$ & $-41,2 \%$ & $45,5 \%$ & $3,9 \%$ & $-57,4 \%$ & $2,0 \%$ & $-28,2 \%$ \\
\hline 5. Infecções de ouvido, nariz e garganta. & $-29,4 \%$ & $80,0 \%$ & $-17,1 \%$ & $7,0 \%$ & $-68,6 \%$ & $-11,1 \%$ & $-43,5 \%$ & $-9,9 \%$ \\
\hline 6. Pneumonias bacterianas & $39,4 \%$ & $16,5 \%$ & $-25,1 \%$ & $-3,8 \%$ & $-19,2 \%$ & $-5,4 \%$ & $-13,0 \%$ & $-0,2 \%$ \\
\hline 7. Asma & $-87,3 \%$ & $-27,0 \%$ & $-55,3 \%$ & $-24,0 \%$ & $-74,0 \%$ & $47,6 \%$ & $-54,1 \%$ & $-34,8 \%$ \\
\hline 8. Doenças pulmonares & $-45,1 \%$ & $-11,0 \%$ & $-44,3 \%$ & $28,4 \%$ & $0,0 \%$ & $-43,6 \%$ & $36,7 \%$ & $10,7 \%$ \\
\hline 9. Hipertensão & $-98,2 \%$ & $-41,2 \%$ & $-52,2 \%$ & $-22,9 \%$ & $-36,4 \%$ & $63,7 \%$ & $-66,7 \%$ & $-33,8 \%$ \\
\hline 10. Angina & \multicolumn{8}{|c|}{ DADOS NÃO DISPONÍVEIS NO SIH/SUS - DATASUS } \\
\hline 11. Insuficiência cardíaca & $-18,5 \%$ & $-28,1 \%$ & $-38,1 \%$ & $-15,7 \%$ & $-40,0 \%$ & $-26,1 \%$ & $-14,1 \%$ & $-16,1 \%$ \\
\hline 12. Doenças cerebrovasculares & $-1,1 \%$ & $0,5 \%$ & $7,3 \%$ & $-13,6 \%$ & $-28,6 \%$ & $-24,1 \%$ & $30,5 \%$ & $15,1 \%$ \\
\hline 13. Diabetes mellitus & $8,2 \%$ & $-2,6 \%$ & $-23,1 \%$ & $-0,7 \%$ & $-5,3 \%$ & $-14,7 \%$ & $-33,5 \%$ & $-20,5 \%$ \\
\hline 14. Epilepsias & $4,5 \%$ & $-50,0 \%$ & $-23,8 \%$ & $1,7 \%$ & $5,6 \%$ & $-1,0 \%$ & $52,9 \%$ & $16,5 \%$ \\
\hline 15. Infecção no rim e trato urinário & $-11,9 \%$ & $-11,0 \%$ & $-39,2 \%$ & $-9,5 \%$ & $-35,8 \%$ & $2,0 \%$ & $-25,7 \%$ & $-15,0 \%$ \\
\hline 16. Infecção da pele e tecido subcutâneo & $173,3 \%$ & $75,0 \%$ & $15,8 \%$ & $65,8 \%$ & $44,9 \%$ & $101,0 \%$ & $-9,5 \%$ & $40,4 \%$ \\
\hline $\begin{array}{l}\text { 17. Doença inflamatória órgãos pélvicos } \\
\text { femininos }\end{array}$ & $-20,0 \%$ & $52,2 \%$ & $-33,3 \%$ & $16,7 \%$ & $-32,8 \%$ & $-65,8 \%$ & $-16,7 \%$ & $-18,2 \%$ \\
\hline 18. Úlcera gastrointestinal & $-83,3 \%$ & $-67,3 \%$ & $-54,8 \%$ & $-58,6 \%$ & $-44,9 \%$ & $-22,2 \%$ & $-29,0 \%$ & $-43,5 \%$ \\
\hline $\begin{array}{l}\text { 19. Doenças relacionadas ao pré-natal e } \\
\text { parto }\end{array}$ & $433,3 \%$ & $-100,0 \%$ & * & $-100,0 \%$ & $425,0 \%$ & $100,0 \%$ & $33,3 \%$ & $53,2 \%$ \\
\hline
\end{tabular}

Fonte: SIH/SUS, 201513.

*Sem dados disponíveis ou frequência igual a zero no ano de 2010.

Outro fato que chamou atenção foi haver ocorrido aumento da variação percentual para doenças preveníveis por imunização e condições sensíveis na maioria das RS. Os maiores incrementos foram observados nas
RS de Quixadá, Limoeiro do Norte, Baturité, Tauá e Iguatu, todos com 100\% ou mais de variação positiva.

É importante destacar algumas limitações presentes neste estudo, a saber: a utilização 
de dados secundários; dados indisponíveis para algumas variáveis analisadas; o uso de projeções populacionais muitas vezes não fidedignas; o registro das internações realizadas apenas no SUS; a possibilidade de ocorrência de eventuais duplicações na contagem de um mesmo paciente; a ausência de dados de morbidade extra-hospitalares; e o fato de a cobertura populacional da ESF ter desconsiderado a (in)adequação da composição das equipes, a qualificação profissional, os vínculos trabalhistas e aspectos estruturais da APS,818-20.

\section{Discussão}

As informações produzidas por este estudo configuram-se úteis para o monitoramento e a avaliação do processo de regionalização em curso no estado, uma vez que a análise das ICSAP pode ser utilizada como parte da avaliação da resolubilidade, da qualidade e do acesso à APS, sobretudo em investigações sobre iniquidades inter-regionais nesse acesso ${ }^{21}$. Pode auxiliar, também, na identificação de necessidades que requeiram a reorientação ou mesmo a proposição de políticas públicas de saúde.

Ressalva-se, contudo, que existem questões que podem refletir no uso inadequado dos serviços de saúde, como particularidades sociodemográficas e epidemiológicas, padrão de uso dos serviços, ausência de rede de proteção social, atitudes individuais frente ao tratamento de doenças, dificuldades de acesso, escassez de recursos, manejo clínico inadequado, fácil acesso à rede especializada e hospitalar e baixo grau de coordenação da APS ${ }^{21}$.

Caminal-Homar \& Casanova-Matutano ${ }^{7}$ afirmam que as ICSAP podem ser evitadas ou diminuídas por ações próprias da APS, seja evitando internações por doenças imunizáveis ou que permitem diagnóstico e tratamento de condições prévias, seja impedindo complicações ou reduzindo as reincidências de internações ao aprimorar o cuidado e o acompanhamento do usuário com problemas crônicos.
Verificou-se no Ceará redução da frequência de ICSAP no período de 2010 a 2014. Os resultados demonstraram que houve uma tendência uniforme para a redução da frequência de ICSAP no período nas RS cearenses, com exceção de Russas e Maracanaú. As reduções nas frequências de internações gerais foram acompanhadas por reduções nas frequências de ICSAP. De forma análoga, as RS de Russas e Maracanaú demonstraram aumento em ambas as frequências, fazendo supor que haja relação direta entre os dois tipos de internação.

Entre as RS do Ceará, a RS de Fortaleza foi a que apresentou o menor percentual de cobertura de EqSF. Ela é composta pelos municípios de Aquiraz, Eusébio, Fortaleza e Itaitinga, e a baixa cobertura na capital cearense interferiu significativamente na cobertura da RS, tendo em vista que os outros municípios atingiram cobertura superior a $90 \%$. Segundo a Sala de Apoio à Gestão Estratégica do Ministério da Saúde, no ano de 2014, a cobertura populacional por EqSF da cidade de Fortaleza foi de 42,05\%, o que corresponde a 1.072.950 habitantes com 311 EqSF. Quanto ao ranking da cobertura populacional nas capitais brasileiras, destacaram-se Teresina (92,39\%), Florianópolis $(89,81 \%)$ e Palmas $(85,61 \%)^{\mathbf{1 0}, 22}$. Pelo baixo percentual de cobertura em Fortaleza, foi estabelecido no Plano Municipal de Saúde de Fortaleza a ampliação de equipes completas da EqSF para $60 \%$ até $2017^{23}$.

O mesmo nexo não foi observado com relação aos coeficientes de internação geral e de ICSAP. Enquanto na RS de Russas houve uma variação percentual positiva para ambos os coeficientes, na RS de Maracanaú houve um aumento apenas no coeficiente de internações gerais. Uma vez que foi observado um aumento nas frequências de internações gerais e por CSAP em ambas as regiões e que os coeficientes são calculados em função da população, isso sugere que esses comportamentos distintos possam estar relacionados às diferenças na velocidade de crescimento populacional nas RS em questão, que, no período analisado, foi bem maior em Maracanaú (variação de 6,2\%) do que em Russas (variação de 3,1\%). 
Na maioria das RS, a ampliação (ou manutenção em 100\%) da cobertura por EqSF esteve acompanhada de uma redução na proporção de ICSAP na comparação entre 2010 e 2014. Essa relação foi observada no estudo de Rehem e Egry $^{3}$ sobre as ICSAP no estado de São Paulo, no período de 2000 a 2007.

Porém, percebe-se que isso não se deu de modo uniforme em todas as RS do Ceará. Tal fato, somado aos casos em que houve, concomitantemente, ampliação da cobertura de EqSF e aumento na proporção de ICSAP, corrobora a hipótese de que uma ampliação da ESF por si, sem o compromisso com a resolubilidade, a qualidade e o acesso, não gera impacto sobre as ICSAP. Ainda que, no período, a ampliação da cobertura tenha contribuído para a redução na proporção de ICSAP de forma geral, pode-se deduzir que as RS cearenses não têm estruturado a APS de modo uniforme, em outras palavras, elas têm demonstrado níveis qualitativos distintos. Salienta-se que a baixa escolaridade, as altas taxas de desemprego, os baixos níveis de renda e menores aportes populacionais têm sido elencados como outros fatores associados a uma maior proporção de ICSAP 24-26.

A análise das ICSAP por grupos de causas nas RS revelou comportamentos diversos. O primeiro ponto que chamou atenção foi o aumento nas ICSAP por doenças preveníveis por imunização e condições sensíveis. Pazó et al. ${ }^{18}$ encontraram o mesmo resultado ao estudarem as ICSAP no estado do Espírito Santo, entre 2005 e 2009. O aumento na cobertura da ESF deveria implicar uma ampliação do acesso à assistência e da prevenção por imunobiológicos, atividade realizada historicamente na APS. Dessa forma, pode-se questionar a efetividade desse procedimento em muitas RS do Ceará no período de estudo. Boing et al. ${ }^{27}$ relatam que, apesar do exemplar programa de imunizações no Brasil, problemas como falta de insumos e de imunobiológicos, deficit de agentes comunitários de saúde nas campanhas de vacinação e resistência dos usuários à vacinação repercutem em deficiência na cobertura vacinal.
As ICSAP por infecções de pele e do tecido subcutâneo foram as que mais aumentaram no estado do Ceará. Sobre essa ampliação temporal da frequência no estado de São Paulo, Rehem e Egry ${ }^{3}$ questionam se o problema reside na organização da ESF para atender a tal diagnóstico ou na baixa sensibilidade desse agravo no nível primário.

As doenças relacionadas ao pré-natal e ao parto demonstraram o segundo maior aumento da frequência de ICSAP no Ceará. O Ministério da Saúde, a partir da Portaria n ${ }^{\circ} 1.459$, de 24 de junho de 2011, lançou a Rede Cegonha, uma rede de atenção à saúde que tem a perspectiva de promover melhoria na assistência à saúde materno-infantil, com focos no parto, nascimento, crescimento e desenvolvimento da criança, garantia do acesso, acolhimento e resolubilidade e redução da mortalidade materna e neonatal ${ }^{28}$. No Ceará, está uma das RAS que mais têm avançado no processo de discussão, planejamento, estruturação e implantação nas RS. É possível que a estruturação prioritária de fluxos de acesso e a ampliação da rede hospitalar vinculada a Rede Cegonha possam corroborar uma ampliação do número de hospitalizações por doenças relacionadas ao pré-natal e ao parto. Contudo, esse panorama também aponta para a necessidade de fortalecimento do componente APS da rede como nível assistencial de promoção, prevenção e tratamento desse grupo de causas.

\section{Conclusões}

A redução das ICSAP no estado do Ceará ao longo dos anos de 2010 a 2014 não deve representar um indicador de qualidade da APS. Em primeiro lugar, por representar um indicador de desempenho indireto e, em segundo lugar, porque o estudo particular de cada RS demonstrou resultados insatisfatórios.

O panorama das ICSAP por grupo de causas sinaliza desafios concretos para a gestão. Questões de saúde envolvendo estratégias de promoção, prevenção e tratamento, que 
podem ser conduzidas em nível primário, evitando hospitalizações desnecessárias, têm demonstrado índices inadequados no cenário estadual, destacando-se as doenças preveníveis por imunização e condições sensíveis, cerebrovasculares e relacionadas ao pré-natal e ao parto. As disparidades entre os resultados verificados nas RS demonstram que elas não têm se desenvolvido de modo uniforme, algumas com baixo desempenho, como no caso da RS de Russas.

Nesse sentido, é importante fortalecer o papel de coordenação da gestão estadual na condução do processo de regionalização. Ampliar e priorizar os espaços de discussão e pactuação, garantir apoio técnico e autonomia às comissões intergestores regionais, estabelecer mecanismos mais eficientes de monitoramento e avaliação de indicadores de saúde e propor políticas de saúde que levem em consideração as distintas realidades regionais podem ser estratégias que colaborem para um melhor desempenho e uma maior uniformidade entre as RS.

O impacto do processo de regionalização da saúde no Ceará na APS nesses últimos anos, embora caminhe para uma redução dos números de ICSAP, parece demonstrar fragmentação do serviço e da gestão, barreiras de acesso e baixas resolubilidade e efetividade das ações em nível primário.
A orientação do sistema de saúde centrado em uma atenção primária de qualidade e resolutiva requer a priorização de investimentos nesse nível assistencial para garantir a efetividade das redes de atenção estruturadas nas RS. Investir na atenção primária pode implicar, em médio e longo prazos, a redução de gastos com procedimentos de alta complexidade e otimização do uso de recursos disponíveis (como leitos hospitalares).

Contudo, espera-se que este trabalho sirva não apenas como referência para a gestão da saúde no estado do Ceará, mas, também, como incentivo ao aprimoramento da análise das ICSAP enquanto um indicador de qualidade indireto da APS.

\section{Colaboradores}

Alves JWS e Cavalcanti CGCS foram responsáveis pela concepção do estudo, pelo levantamento, pela análise e interpretação dos dados e pela redação do artigo. Alves RSM e Costa PC participou da análise e da interpretação dos dados e da revisão crítica do artigo. Todos os autores aprovaram a versão final e se responsabilizam por todos os aspectos do trabalho, incluindo a garantia de sua precisão e integridade. 


\section{Referências}

1. Starfield B. Atenção primária: equilíbrio entre necessidades de saúde, serviços e tecnologia. Brasília, DF: Ministério da Saúde; 2002.

2. Assis MMA, Jesus WLA. Acesso aos serviços de saúde: abordagens, conceitos, políticas e modelo de análise. Ciênc. Saúde Colet. 2012; 17(11):2865-2875.

3. Rehem TCMSB, Egry EY. Internações por condições sensíveis à atenção primária no estado de São Paulo. Ciênc. Saúde Colet. 2011; 16(12):4755-4766.

4. Brasil. Ministério da Saúde. Portaria $n^{\circ} 4.279$, de 30 de dezembro de 2010. Estabelece diretrizes para a organização da Rede de Atenção à Saúde no âmbito do Sistema Único de Saúde (SUS). Diário Oficial da União. 31 Dez 2010.

5. Brasil. Ministério da Saúde. Política Nacional de Atenção Básica. Brasília, DF: Ministério da Saúde, 21 Out 2011.

6. Souza LL, Costa, JSD. Internações por condições sensíveis à atenção primária nas coordenadorias de saúde no RS. Rev. Saúde Pública. 2011; 45(4):765-772.

7. Caminal-Homar J, Casanova-Matutano C. La evaluación de la atención primaria y las hospitalizaciones por ambulatory care sensitive conditions. Marco conceptual. Atención Primaria. 2003; 31(1);61-65.

8. Macinko J. Estados Unidos: estudo da lista de Internações por condições sensíveis à atenção básica. In: Relatório de Atividades do III Seminário Internacional Atenção Primária Saúde da Família: expansão com qualidade e valorização de resultados. [internet]. 2007 dez 13-15 [acesso em 2015 mar 12]; Brasília, DF: Ministério da Saúde; 2008. p. 186-192. Disponível em: http://189.28.128.100/dab/docs/publicacoes/geral/3seminario_internacional_relatorio_atividades.pdf.

9. Brasil. Ministério da Saúde. Portaria nº 221, de 17 de abril de 2008. Publica na forma de anexo a Lista
Brasileira de Internações por Condições Sensíveis à Atenção Primária. Diário Oficial da União. 18 Abr 2008.

10. Ceará. Secretaria da Saúde do Estado. [internet] Revisão do Plano Diretor de Regionalização das Ações e Serviços de Saúde - PDR do Estado do Ceará 2014. [acesso em 2015 mar 12]. Disponível em: http://www. saude.ce.gov.br/index.php/downloads/category/99-plano-diretor-de-regionalizacao-pdr-2014.

11. Brasil. Decreto $n^{\circ} 7.508$, de 28 de junho de 2011. Regulamenta a Lei no 8.080, de 19 de setembro de 1990, para dispor sobre a organização do Sistema Único de Saúde - SUS, o planejamento da saúde, a assistência à saúde e a articulação interfederativa, e dá outras providências. Diário Oficial da União. 29 Jun 2011.

12. Secretaria da Saúde do estado do Ceará. [internet] Coordenadorias regionais de saúde. [acesso em 2015 mar 13]. Disponível em: http://www.saude.ce.gov.br/ index.php/regionalizacao.

13. Brasil. Ministério da Saúde. Departamento de Informática do SUS. [internet] Morbidade hospitalar do SUS - por local de residência - Ceará. [acesso em 2015 mar 15]. Disponível em: http://tabnet.datasus. gov.br/cgi/deftohtm.exe?sih/cnv/nrce.def.

14. Brasil. Ministério da Saúde. Departamento de Atenção Básica. [internet] Histórico de cobertura da saúde da família. [acesso em 2015 mar 13]. Disponível em: http://dab.saude.gov.br/portaldab/historico_cobertura_sf.php.

15. Brasil. Ministério da Saúde. Departamento de Atenção Básica. [internet] Sistema de Informação da Atenção Básica - SIAB. [acesso em 2015 mar 12]. Disponível em: http://dab.saude.gov.br/portaldab/siab.php.

16. Brasil. Ministério da Saúde. Departamento de Atenção Básica. [internet] Histórico de cobertura da saúde da família: nota técnica. [acesso em 2015 mar 12]. Disponível em: http://189.28.128.100/dab/docs/ge- 
ral/historico_cobertura_sf_nota_tecnica.pdf.

17. Instituto Brasileiro de Geografia e Estatística. [internet]. Censo demográfico 2010. [acesso em 2015 mar 14]. Disponível em: https://censo2010.ibge.gov.br/ resultados.html.

18. Pazó RG, Frauches DO, Galvêas DP, et al. Internações por condições sensíveis à atenção primária no Espírito Santo: estudo ecológico descritivo no período 2005-2009. Epidemiol. Serv. Saúde. 2012; 21(2):275282.

19. Rodrigues-Bastos RM, Campos EMS, Ribeiro LC, et al. Internações por condições sensíveis à atenção primária em município do sudeste do Brasil. Rev. Assoc. Med. Bras. 2013; 59(2):120-127.

20. Escrivão Júnior A. Internações por condições sensíveis à atenção primária na Região Metropolitana de São Paulo. São Paulo: Fundap, 2014.

21. Alfradique ME, Bonolo PF, Dourado I, et al. Internações por condições sensíveis à atenção primária: a construção da lista brasileira como ferramenta para medir o desempenho do sistema de saúde (Projeto ICSAP - Brasil). Cad. Saúde Pública. 2009. 25(6):13371349.

22. Brasil. Ministério da Saúde. Sala de Apoio à Gestão Estratégica [internet] [acesso em 2018 jan 25]. Disponível em: http://sage.saude.gov.br.

23. Fortaleza. Secretaria Municipal de Saúde. Plano Municipal de Saúde de Fortaleza 2014 - 2017 [internet] [acesso em 2018 jan 25]. Fortaleza: Secretaria Municipal de Saúde; 2017. Disponível em: https://saude. fortaleza.ce.gov.br/infosaude-menu/67-plano-municipal-de-saude-2.

24. Márquez-Calderón S, Águila MMR, Perea-Milla E, et al. Factores asociados a la hospitalizacion por procesos sensibles a cuidados ambulatorios en los municipios. Gac Sanit. 2003; 17(5):360-367.

25. Roos LL, Wald R, Uhanova J, et al. Physician visits, hospitalizations, and socioeconomic status: ambulatory care sensitive conditions in a canadian setting. Health Serv Res. 2005; 40(4):1167-1185.

26. Dias-Da-Costa JS, Büttenbender DC, Hoefel AL, et al. Hospitalizações por condições sensíveis à atenção primária nos municípios em gestão plena do sistema no estado do Rio Grande do Sul, Brasil. Cad. Saúde Pública. 2010; 26(2):358-364.

27. Boing AF, Vicenzi RB, Magajewski F, et al. A. Redução das internações por condições sensíveis à atenção primária no Brasil entre 1998-2009. Rev Saúde Pública. 2012. 46(2):359-366.

28. Brasil. Ministério da Saúde. Portaria n ${ }^{\circ} 1.459$, de 24 de junho de 2011. Institui, no âmbito do Sistema Único de Saúde - SUS -, a Rede Cegonha. Diário Oficial da União. 27 Jun 2011.

Recebido em 21/09/2017

Aprovado em 29/01/2018

Conflito de interesses: inexistente

Suporte financeiro: não houve 